\title{
SARS-CoV-2 transmission rate is low when following a COVID+ patient in the operating room
}

\author{
Lucas G. Axiotakis Jr. ${ }^{1}$. Deborah M. Boyett ${ }^{2} \cdot$ Brett E. Youngerman ${ }^{2} \cdot$ Guy M. McKhann ${ }^{2} \cdot$ Anil K. Lalwani ${ }^{3,4}$ (I)
}

Received: 9 October 2020 / Accepted: 10 January 2021 / Published online: 6 February 2021

(C) The Author(s), under exclusive licence to Springer-Verlag GmbH, DE part of Springer Nature 2021

\begin{abstract}
Purpose Acquiring SARS-CoV-2 infection for uninfected patients undergoing surgical procedures following a COVID positive (COVID+) patient is of significant concern, both for patients seeking medical care in hospital settings and for management of surgical services during pandemic times.

Methods Using data identifying all COVID+ surgical patients during the initial pandemic peak in New York City (March 15 to May 15, 2020), we analyzed the rate of postoperative symptomatic SARS-CoV-2 infection in COVID negative (COVID-) patients undergoing surgery in the same operating room within $48 \mathrm{~h}$, thus determining nosocomial symptomatic infection rate attributable to COVID operating room exposure.

Results Five COVID- patients directly followed a COVID+ patient, while 19 patients were exposed to COVID+ operating rooms within 24 h. By 48 h, 21 additional patients were exposed. No exposed patients acquired symptomatic SARS-CoV-2 infection postoperatively.

Conclusion With implementation of infection prevention and control procedures in the operating room under local pandemic conditions, our findings suggest that the risk of acquiring SARS-CoV-2 infection, when following a COVID+ patient in the same operating room, is very low.
\end{abstract}

Keywords Nosocomial infection $\cdot$ COVID-19 pandemic $\cdot$ Patient safety $\cdot$ Perioperative services

Drs. McKhann and Lalwani share senior authorship for this work.

Previous use: The definitions and identification of patient subpopulations based on pre- and post-operative COVID status were used in another study by our group (PMID: 33156061) examining perioperative SARSCoV-2 infection and was carried forward for the purposes of the current work focusing on transmission due to operating room exposure.

Anil K. Lalwani

ak12144@cumc.columbia.edu

1 Columbia University Vagelos College of Physicians and Surgeons, New York, NY, USA

2 Department of Neurological Surgery, New York-Presbyterian Hospital, Columbia University Irving Medical Center, New York, NY, USA

3 Department of Otolaryngology—Head and Neck Surgery, New York-Presbyterian Hospital, Columbia University Irving Medical Center, Columbia Vagelos College of Physicians and Surgeons, 180 Fort Washington Avenue, Harkness 8, New York, NY 10032, USA

4 Department of Mechanical Engineering, The Fu Foundation School of Engineering and Applied Science, Columbia University, New York, NY, USA

\section{Introduction}

During the current global coronavirus disease 2019 (COVID19) pandemic, acquiring SARS-CoV-2 infection in the hospital is of significant concern for patients needing acute care. In the operating room (OR), aerosolized SARS-CoV-2 particles and those surviving on surfaces for extended periods $(>48 \mathrm{~h})$ are worrisome for nosocomial transmission [1-3]. This is particularly concerning for COVID negative (COVID-) patients undergoing invasive surgical procedures immediately following COVID positive (COVID+) patients in the same OR.

To minimize risk of SARS-CoV-2 transmission between surgical cases during the pandemic, hospitals have implemented infection prevention and control procedures specific to the $\mathrm{OR}$ environment, including designating COVID ORs, instituting routine "terminal" cleaning of the OR (i.e., protocols normally performed at the end of the day including disinfecting all vertical and horizontal surfaces, equipment, and receptacles), delaying entry to the room by 30 -minutes to effect $>99.9 \%$ air turnover, and placing viral filters in the expiratory limb of the circuit and between the breathing circuit and the patient's airway to prevent 
Table 1 Nature of perioperative COVID exposures

\begin{tabular}{lllll}
\hline COVID+ OR exposures* & \multicolumn{4}{l}{ Testing status of COVID+ OR patient $(N)^{* *}$} \\
\cline { 2 - 5 } & Presumed positive & $\begin{array}{l}\text { Positive test } \\
1-3 \text { days preop }\end{array}$ & $\begin{array}{l}\text { Positive test }>3 \\
\text { days preop }\end{array}$ & Total \\
\hline Immediately following COVID+ case & 3 & 0 & 2 & 5 \\
Exposure within 24 h of COVID+ case & 7 & 2 & 14 & 23 \\
Exposure 24-48 h after COVID+ case & 10 & 9 & 10 & 29 \\
Total & 20 & 11 & 26 & 57 \\
\hline
\end{tabular}

Table 1 includes all individual instances of exposures to specific COVID+ ORs $(n=57)$. Number of patients exposed $(n=45)$ is not shown; this difference is accounted for by $n=9$ patients who were exposed to COVID+ ORs on $>1$ occasion within $48 \mathrm{~h}$

*Time elapsed between a COVID+ patient undergoing surgery followed by a COVID- in the same OR was stratified by (row 1) the next case in the COVID+ OR within the same day, (row 2) within $24 \mathrm{~h}$ but not directly following a COVID+ cases within the same day, and (row 3) within $24-48 \mathrm{~h}$ of a COVID+ case

**Preoperative COVID+ patients were stratified by (column 1) remote positive testing $>3$ days preoperatively; (column 2) positive test within 0 to 3 days preoperatively; (column 3) presumed infection based on 2+ of 7 CDCdefined symptoms without testing or with positive test on postoperative day 1 [4] transmission via ventilators. Despite these measures, their effectiveness in preventing transmission due to OR exposure is unclear. Herein, we investigate the risk of acquiring perioperative SARS-CoV-2 infection when a COVID- patient follows a COVID+ patient in the same OR.

\section{Materials and methods}

All index surgeries occurring at Columbia University during the initial peak of the COVID-19 pandemic, from
March 15 to May 15, 2020, were analyzed. Preoperatively and postoperatively, patients were considered COVID+ if they tested positive or had 2+ of 7 symptoms based on Centers for Disease Control (CDC) guidelines [4]. Exposure to SARS-CoV-2 was defined as surgery occurring after a COVID+ patient in the same OR up to $48 \mathrm{~h}$ postoperatively; therefore, a COVID- patient could undergo surgery in the same operating room as no COVID+ patients, one COVID+ patient, or greater than one COVID+ patient within this timeframe. "Exposure" for the COVID- patient was defined as the number of
Fig. 1 COVID- surgical patients exposed to COVID+ ORs within $48 \mathrm{~h}$, March 15-May 15. $X$-axis $=$ study week ( 7 days each beginning on March 15). Week 9 consists of only 6 days due to the study period end date (ending on May 15). Left $Y$-axis (bars) $=$ total number of exposures per week of COVID- patients to ORs in which a COVID+ patient had undergone surgery within $48 \mathrm{~h}$. The key indicates screening status of COVID+ patients (presumed positive based on symptoms, or positive testing preoperatively) accounting for OR exposures. Right $Y$-axis (lines) = weekly averages of daily case counts (diamonds) and number of ORs in use (squares) during the study period, representing the initial peak of the pandemic at our tertiary hospital center

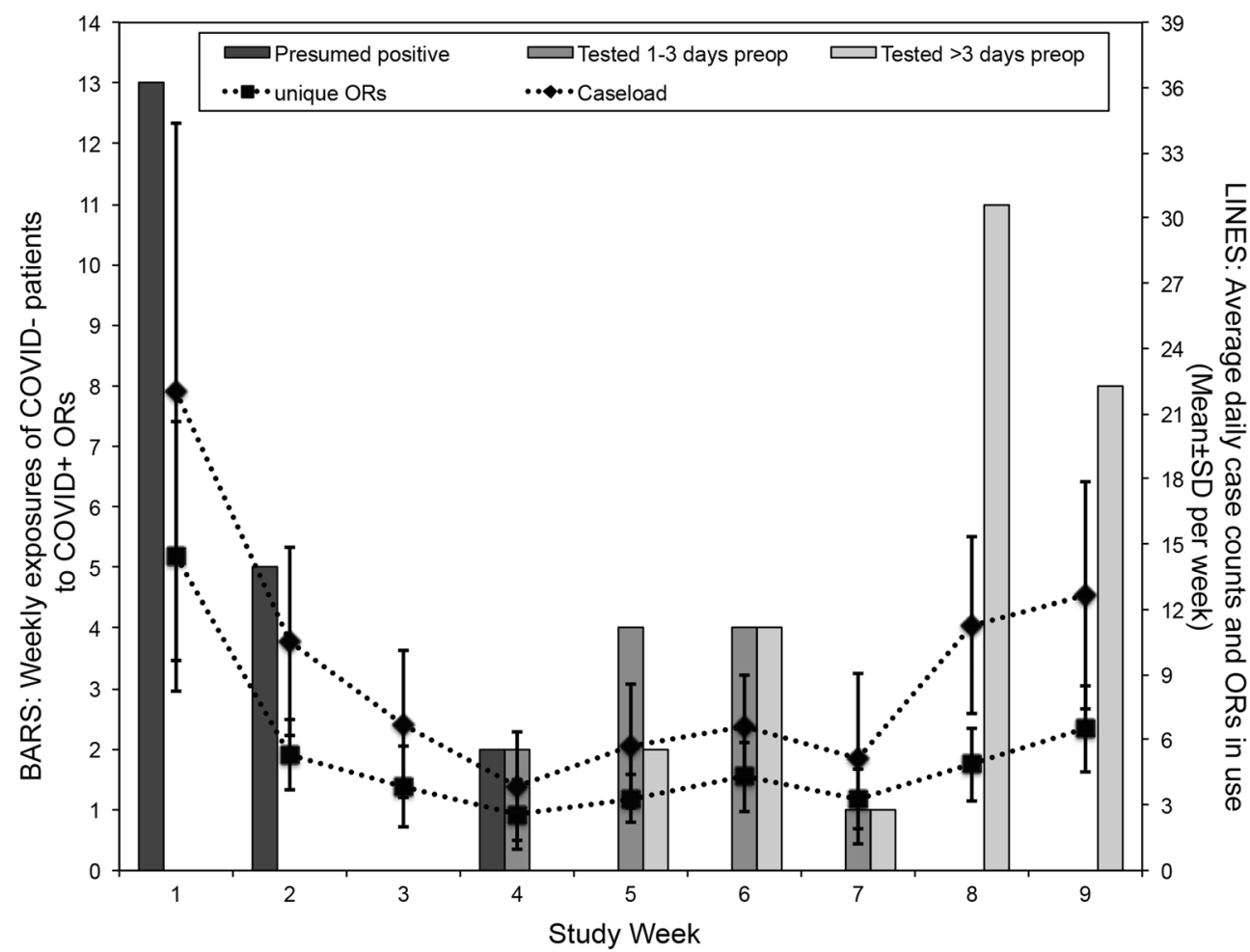


COVID+ cases conducted in the previous $48 \mathrm{~h}$ in the same OR. The Columbia University Institutional Review Board approved the collection of the data analyzed in this study.

\section{Results}

During the study time period, 552 analyzable patients (51 COVID+ and 501 COVID-) underwent surgery. Five COVID - patients (1.0\%) directly followed a COVID+ case on the same day. Nineteen patients $(3.8 \%)$ went on to follow a COVID+ case, resulting in 23 exposures, within $24 \mathrm{~h}$ (Table 1); none acquired symptomatic SARS-CoV2 infection postoperatively. Twenty-one COVID- patients $(4.2 \%)$ followed a COVID+ case within 24 and $48 \mathrm{~h}$, resulting in 29 exposures; similarly, none acquired symptomatic SARS-CoV-2 infection postoperatively. Nine patients (1.8\%) followed more than one COVID+ patient undergoing surgery in the same OR within the prior $48 \mathrm{~h}$. During the same study period, 9 (of 456, or 1.9\%) patients without COVID+ OR exposure acquired postoperative SARS-CoV-2 infection confirmed by PCR testing. Exposure to COVID+ ORs was not significantly associated with symptomatic perioperative SARS-CoV-2 infection (Fisher's exact test, $p=1.00$ ). Total weekly exposures of COVID - patients to COVID+ ORs correlated with average caseload and the number of ORs in use as the study period unfolded (Fig. 1).

\section{Discussion}

With continued increases in hospitalized COVID-19 patients throughout various areas of the USA, hospitalacquired SARS-CoV-2 infection remains a significant concern. In addition to OR-specific safety measures, surgical services have adopted a multitude of approaches aimed at reducing nosocomial infection, including but not limited to performing emergent procedures only, decreasing overall caseload and ORs in use, and instituting mandatory screening to inform triage and preoperative decision-making [5, 6]. Nonetheless, on occasion, when a COVID- patient follows in a room where a COVID+ patient underwent surgery, transmission concerns remain high. In this study, we show that, under these circumstances, the risk of symptomatic COVID infection is very low. Our finding suggests that the infection prevention and control procedures implemented within ORs have been effective in minimizing COVID transmission among surgical patients; this finding may help alleviate patient concerns of nosocomial SARSCoV-2 infection, especially when inpatient and surgical care is necessary during the pandemic. Ostensibly, exposure to COVID of the small cohort of patients acquiring symptomatic infection perioperatively occurred in other areas of the hospital environment, or in the community at large. This study is limited by its retrospective nature and reliance on chart review for diagnosis, evolution of protocols during the study period, and inability to assess the COVID status of OR staff.

\section{Conclusion}

With thorough implementation of infection prevention and control procedures in the OR, our findings suggest that the risk of symptomatic infection following a COVID+ patient in the same OR is very low.

Acknowledgements The authors wish to acknowledge Randy Casals, MD (Columbia University Irving Medical Center-New YorkPresbyterian Hospital), Tyler Cooke, MD (Columbia University Irving Medical Center-New York-Presbyterian Hospital), Graham Winston, MD (New York-Presbyterian Hospital), and Cory Chang, AB (Columbia University College of Physicians and Surgeons), for contributions to data acquisition and statistical guidance.

Author contribution AKL and GMM conceived of the study. AKL, GMM, LGA, DMB, and BEY performed data collection, analysis, and interpretation. All authors contributed meaningfully to and approved of the final manuscript.

Availability of data and material The de-identified data analyzed in this study is available upon reasonable request.

Code availability Not applicable.

\section{Declarations}

Ethics approval The data analyzed in this study was collected as part of Columbia University Institutional Review Board protocol \#AAAT0618.

Conflict of interest The authors declare that they have no conflict of interest.

\section{References}

1. Zakka K, Erridge S, Chidambaram S, Kynoch M, Kinross J, Purkayastha S (2020) Electrocautery, diathermy, and surgical energy devices: are surgical teams at risk during the COVID-19 pandemic? Ann Surg. https://doi.org/10.1097/SLA.0000000000004112

2. Suman R, Javaid M, Haleem A, Vaishya R, Bahl S, Nandan D (2020) Sustainability of coronavirus on different surfaces. J Clin Exp Hepatol. https://doi.org/10.1016/j.jceh.2020.04.020

3. van Doremalen N, Bushmaker T, Morris DH et al (2020) Aerosol and surface stability of SARS-CoV-2 as compared with SARS-CoV1. N Engl J Med. https://doi.org/10.1056/NEJMc2004973

4. (2020) Symptoms of Coronavirus (COVID-19). Centers for Disease Control website. https://www.cdc.gov/coronavirus/2019-ncov/ downloads/COVID19-symptoms.pdf. Accessed 15 Jun 2020 
5. COVIDSurg Collaborative (2020) Global guidance for surgical care during the COVID-19 pandemic. Br J Surg. https://doi.org/10.1002/ bjs. 11646

6. Ross SW, Lauer CW, Miles WS, Green JM, Christmas AB, May AK et al (2020) Maximizing the calm before the storm: tiered surgical response plan for novel coronavirus (COVID-19). J Am Coll Surg. https://doi.org/10.1016/j.jamcollsurg.2020.03.019

Publisher's note Springer Nature remains neutral with regard to jurisdictional claims in published maps and institutional affiliations. 